\title{
Intravenous High-Dose and Supplementation of Vitamin C Can Effectively Prevent and Treat COVID-19?
}

\author{
Attapon Cheepsattayakorn ${ }^{1,2 *}$, Ruangrong Cheepsattayakorn ${ }^{3}$ and \\ Utoomporn Sittisingh ${ }^{2}$ \\ ${ }^{1} 10^{\text {th }}$ Zonal Tuberculosis and Chest Disease Center, Chiang Mai, Thailand \\ ${ }^{2} S t$. Theresa International College, Nakorn Nayok, Thailand \\ ${ }^{3}$ Department of Pathology, Faculty of Medicine, Chiang Mai University, Chiang Mai, \\ Thailand \\ *Corresponding Author: Attapon Cheepsattayakorn, $10^{\text {th }}$ Zonal Tuberculosis and \\ Chest Disease Center, Changklan, Muang, Chiang Mai, Thailand.
}

Coronaviruses and influenza viruses, the pandemic viruses can cause acute respiratory distress syndrome (ARDS) contributing to lethally acute lung injuries (ALI) and death. Activation of nuclear factor erythroid 2 (nfe2)-related factor 2 (nrf2), a major regulator of antioxidant response element (ARE)-driven cytoprotective protein expression plays an critical role in preventing cells and tissues from oxidative-stress-induced injury. Both viral and bacterial infections can contribute to cytokine storm that results in increased oxidative stress. Vitamin $\mathrm{C}$ is beneficial to critical care management due to its important component of the cellular antioxidant system. High-dose vitamin $\mathrm{C}$ has been clinically used for several decades. Intravenous high dose of vitamin C may be applicable to COVID-19 since the prevention and management of oxidative stress could be administered by large dose of antioxidant. By eating a variety of food, particularly fruits and vegetables, individuals can easily achieve 100 to $200 \mathrm{mg}$ of vitamin $\mathrm{C}$ that is proved to be adequate to optimize cell and tissue levels for the reduction of the risks of chronic diseases. One medium-size orange contains 70 - 80 mg of vitamin C, one cup (around 100 grams) of broccoli contains $90 \mathrm{mg}$ of vitamin $\mathrm{C}$, and one large red guava contains approximately $120 \mathrm{mg}$ of vitamin C. At least $200 \mathrm{mg}$ of daily vitamin $\mathrm{C}$ supplementation is effective in improving the duration and severity of the common cold, both in children and adults. Nevertheless, the "prophylactic" dose of vitamin C for disease prevention is controversial.

A clinical trial in 50 patients with moderate to severe COVID-19 in China revealed that the dose used varied between $2 \mathrm{~g}$ and $10 \mathrm{~g}$ daily, given over the period of 8 to 10 hours. Among patients with critical condition, additional vitamin $\mathrm{C}$ bolus may be required. Improving oxygenation index in real time was achieved and finally, all patients were discharged. A recent United States National Institute of Health (NIH) expert panel document demonstrated clearly that the regimen of $1.5 \mathrm{~g} / \mathrm{kg}$ body weight is safe and without significant adverse side-effects. Another clinical trial reported that various high-dose intravenous vitamin C infusion $(200 \mathrm{mg} / \mathrm{kg}$ body weight daily, divided into 4 doses, etc.) shortened the intensive care unit (ICU) stay by $97.8 \%$, in addition to a significant reduction in the ICU mortality rate as well as patients with severe influenza. Additionally, oral vitamin C (6g daily, etc.) was able to reduce viral infection risk or to improve clinical symptoms. A recently published randomized-clinical-trial conducted in the USA among 167 patients with sepsis-related ARDS demonstrated that intravenous administration of around $15 \mathrm{~g}$ daily of vitamin $\mathrm{C}$ for 4 days may reduce the mortality. A clinical trial of ICU-admitted patients with pneumonia and earlier intravenous administration of vitamin C included hydrocortisone administration. Nevertheless, systemic corticosteroid administration has not been demonstrated to have significant benefit in COVID-19 patients. Recently, a clinical trial on investigation of vitamin C infusion (a dose of $24 \mathrm{~g}$ daily for 7 days) for the treatment of 140 patients with severe COVID-19 pneumonia was registered on clinicaltrials.gov (Identifier: NCT04264533) has started in Wuhan city, China. These investigators will assess the ICU length of stay, requirements for mechanical ventilation and vasopressor drugs, organ failure scores, and 28-day mortality. This trial is expected to be complete by the end of September 2020. Vitamin D receptors on the immune cells also affect their function indicating profoundly influences individuals' response to infections. Some researchers are urging individuals to take daily 2,000 international units (IUs) of vitamin D, rather than the recommended 400 to 800 IUs that depends on age.

In conclusion, although the findings of several studies on vitamin $\mathrm{C}$ or other vitamins or other antioxidants in preventing and treating patients with COVID-19 will be too late for current patients with COVID-19, these studies will however provide valuable information during future viral outbreaks. Clearly, 
well-designed clinical trials are completely and urgently needed to develop bedside-use standard protocols.

\section{Assets from publication with us}

- Prompt Acknowledgement after receiving the article

- Thorough Double blinded peer review

- Rapid Publication

- Issue of Publication Certificate

- High visibility of your Published work

Website: www.actascientific.com/

Submit Article: www.actascientific.com/submission.php Email us: editor@actascientific.com

Contact us: +919182824667 